\title{
Converging evidence that short-active photoperiod increases acetylcholine signaling in the hippocampus
}

\author{
Zackary A. Cope ${ }^{1,2} \cdot$ Maria L. Lavadia ${ }^{1} \cdot$ Aniek J. M. Joosen $^{3} \cdot$ Chuck J. A. van de Cappelle ${ }^{3} \cdot$ Joseph C. Lara ${ }^{1}$. \\ Alexandra Huval ${ }^{1}$ • Molly K. Kwiatkowski ${ }^{1}$. Marina R. Picciotto ${ }^{4} \cdot$ Yann S. Mineur ${ }^{4}$ - Davide Dulcis ${ }^{1}$. \\ Jared W. Young ${ }^{1,5}$
}

Published online: 13 August 2020

(C) The Psychonomic Society, Inc. 2020

\begin{abstract}
Seasonal variations in environmental light influence switches between moods in seasonal affective disorder (SAD) and bipolar disorder (BD), with depression arising during short active (SA) winter periods. Light-induced changes in behavior are also seen in healthy animals and are intensified in mice with reduced dopamine transporter expression. Specifically, decreasing the nocturnal active period (SA) of mice increases punishment perseveration and forced swim test (FST) immobility. Elevating acetylcholine with the acetylcholinesterase inhibitor physostigmine induces depression symptoms in people and increases FST immobility in mice. We used SA photoperiods and physostigmine to elevate acetylcholine prior to testing in a probabilistic learning task and the FST, including reversing subsequent deficits with nicotinic and scopolamine antagonists and targeted hippocampal adeno-associated viral administration. We confirmed that physostigmine also increases punishment sensitivity in a probabilistic learning paradigm. In addition, muscarinic and nicotinic receptor blockade attenuated both physostigmine-induced and SA-induced phenotypes. Finally, viral-mediated hippocampal expression of human AChE used to lower ACh levels blocked SA-induced elevation of FST immobility. These results indicate that increased hippocampal acetylcholine neurotransmission is necessary for the expression of SA exposure-induced behaviors. Furthermore, these studies support the potential for cholinergic treatments in depression. Taken together, these results provide evidence for hippocampal cholinergic mechanisms in contributing to seasonally depressed affective states induced by short day lengths.
\end{abstract}

Keywords Bipolar disorder $\cdot$ Bipolar depression $\cdot$ Seasonal affective disorder $\cdot$ Acetylcholine $\cdot$ Scopolamine $\cdot$ Mecamylamine

Seasonal factors inducing state-dependent affect and relapse were first described in bipolar disorder (BD) patients (Geoffroy et al., 2015). During the spring and summer, in which there is a longer light (active) period, BD patients exhibit more frequent manic episodes. These individuals exhibit

Jared W. Young

jaredyoung@ucsd.edu

1 Department of Psychiatry, University of California San Diego, 9500 Gilman Drive MC 0804, La Jolla, CA 92093-0804, USA

2 Present address: University of Pittsburgh Aging Institute, Pittsburgh, PA, USA

3 Division of Pharmacology, Utrecht Institute for Pharmaceutical Sciences, Utrecht University, Utrecht, The Netherlands

4 Department of Psychiatry, Yale School of Medicine, New Haven, CT, USA

5 Research Service, VA San Diego Healthcare System, San Diego, CA, USA more frequent depressive episodes, however, as light availability and activity decreases in autumn and winter (Barbini, Di Molfetta, Gasperini, Manfredonia, \& Smeraldi, 1995; Geoffroy et al., 2015; Lee, Tsai, \& Lin, 2007; Mulder, Cosgriff, Smith, \& Joyce, 1990; Parker \& Walter, 1982; Sayer, Marshall, Mellsop, 1991; Takei et al., 1992; Volpe \& Del Porto, 2006; Wang \& Chen, 2013). Treatments seeking to restore and maintain stability of photoperiod (timed light exposure or phototherapy, social rhythm therapy) can support a euthymic (asymptomatic) state (Postolache \& Oren, 2005; Wu et al., 2009), while minimizing light exposure using blue lightblocking glasses can reduce manic symptoms when used as adjunctive therapy (Saricicek et al., 2013; Young \& Dulcis, 2015). Similar patterns of light exposure can influence measures relevant to depression and mania in experimental animals as well (Dulcis, Jamshidi, Leutgeb, \& Spitzer, 2013; Fernandez et al., 2018; Young et al., 2018). Shorter active photoperiods (SAP) may therefore serve as an environmental trigger for the induction of depression in BD (Young \& 
Dulcis, 2015), and particularly in seasonal affective disorder (SAD; Parry \& Maurer, 2003; Partonen \& Lonnqvist, 1998; Rosenthal et al., 1984), wherein winter photoperiods drive depressive episodes. Among the adaptations induced by SAP exposure, it has been proposed that a molecular cascade results in elevated acetylcholine (ACh) levels in the brain, which is a mechanism relevant to depression (AshkenazyFrolinger, Kronfeld-Schor, Juetten, \& Einat, 2010; Dulcis et al., 2013; Young \& Dulcis 2015), consistent with elevated ACh in people with depression (Hannestad et al., 2013), but confirmation is required.

SAP exposure induces "re-specification" of neurotransmitter phenotype in the medial hypothalamus that is associated with elevated levels of circulating corticotropin releasing factor (CRF). This switch can in turn modulate circulating corticosteroids, driving the stress response and depression (Dulcis et al., 2013). Interestingly, CRF expression elevates ACh, particularly in the hippocampus (Desvignes et al., 2003). Pharmacological increase of ACh in human subjects, via blockade of AChesterase (AChE; the primary enzyme mediating ACh degradation) by physostigmine, acutely induces clinically relevant symptoms of depression in healthy people (Risch et al., 1981), and euthymic BD patients (Shopsin, Janowsky, Davis, \& Gershon, 1975). Some participants in these studies reached severity that would typically warrant inpatient hospitalization. These symptoms were rapidly reversed by administration of the antimuscarinic acetylcholine receptor (mAChR) drug atropine (Janowsky, el-Yousef, Davis, \& Sekerke, 1973; Risch et al., 1981). Notably, physostigmine treatment also alleviated manic symptoms in patients with $\mathrm{BD}$, although depressed episodes often followed (Janowsky, el-Yousef, Davis, Hubbard, Sekerke, 1972; Risch et al., 1981). In rodents, physostigmine increased immobility of mice in the forced swim test (FST; Mineur et al., 2013), that was reversed by lithium (van Enkhuizen, Milienne-Petiot, Geyer, \& Young, 2015). Because immobility in the FST is a relevant measure to detect antidepressant efficacy and the neurobiological systems relevant for stress adaptation, but does not specifically represent symptoms of depression, additional evidence is needed to determine whether physostigmine recreates other aspects of depression (e.g., punishment sensitivity; Young et al., 2018), and the mechanism underlying these effects.

The present study focused on determining the mechanistic relationship between SAP exposure and $\mathrm{ACh}$ in the induction of immobility in FST as a proxy for mechanisms underlying stress reactivity. While previous findings have clearly implicated hippocampal acetylcholine in the induction of FST immobility (Mineur et al., 2013), the link between SAP and ACh has yet to be determined. These experiments also sought to determine this relationship at the level of the hippocampus, as well as the relative contributions of the two primary sources of cholinergic activity effects, $\mathrm{mAChRs}$ and nicotinic $\mathrm{ACh}$ receptors (nAChR) as a mechanism relevant to depression resulting from SAP exposure.

\section{Method}

\section{Subjects}

Adult male and female C57BL/6J mice, ages 3-6 months, were obtained from Jackson Laboratories and used for all experiments. All animals were housed 3-4 per cage in a temperature and humidity-controlled vivarium in a facility at University of California San Diego (UCSD) accredited by Association for the Assessment and Accreditation of Laboratory Animal Care (AAALAC). Unique cohorts were used for each individual experiment described below. For experiments without photoperiod manipulation, animals were housed in a reversed 12-hour light cycle (lights on at 7:00 PM and off at 7:00 AM). Mice in Experiment $3 \mathrm{a}$ were housed in shorter photoperiod chambers (four cages per chamber). For all other photoperiod experiments, animals were housed in one of 10 identical custom chambers (six cages per chamber) within a vivarium room. All chambers were individually ventilated and illuminated to 130 lumens, measured in the center of the chamber, by three horizontal, programmable white LED strips equally spaced vertically on each side wall. Animals were housed either in SAP (19 hours light : 5 hours dark) or normal active photoperiod (NAP; 12 hours light : 12 hours dark) with the start of the active period (dark) temporally aligned for both conditions. All animals were tested during their common active phase of the light cycle. Mice had ad libitum access to water and food. All procedures were approved by the UCSD Institutional Animal Care and Use Committee.

\section{Probabilistic reversal learning task (PRLT)}

The PRLT is a rodent operant task used to measure reward and punishment learning contingencies as well as behavioral flexibility when these reward/punishment contingencies are switched (Amitai et al., 2014; Milienne-Petiot et al., 2018). In short, the animal must choose between two differentially rewarded options, one with a high probability of reward (80:20) and another with a low probability (20:80). After making eight consecutive choices of the high probability option, the contingencies are reversed, quantified as switches (depicted in Fig. 1a). The discrete trial structure of the task also enables examination of behavior following reward or punishment feedback resulting from choices on preceding trials. Specifically, an increase in sensitivity to punishment, a clinically relevant measure of depression, manifests as an increase in lose-shift behavior (making a different choice from the previous trial after a punishment). 

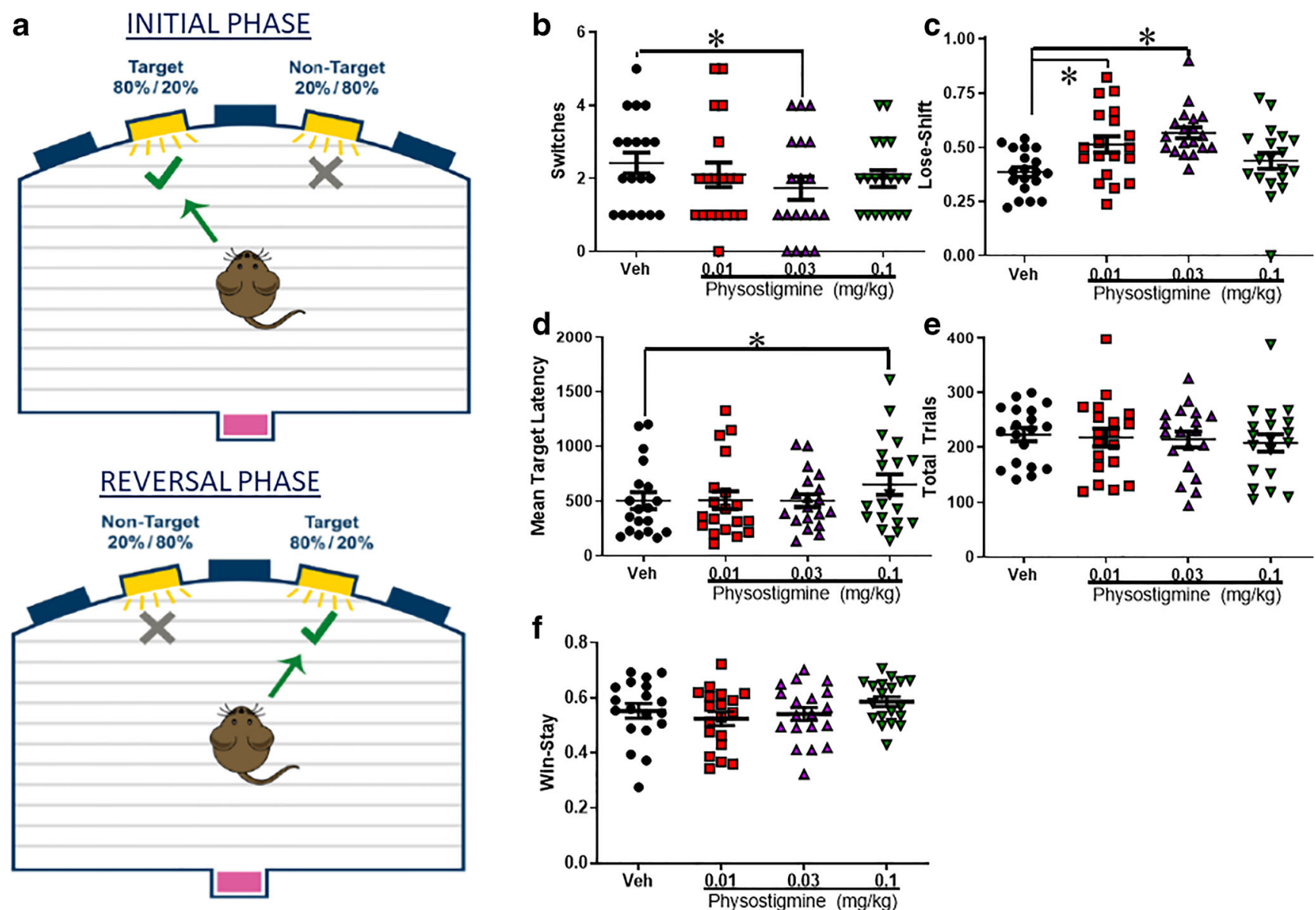

Fig. 1 Physostigmine induced cognitive inflexibility driven by increasing punishment sensitivity. Female and male C57BL/6J mice ( $n=10$ and 9, respectively) were trained in the probabilistic reversal learning task (PRLT; a). The effects of the acetylcholinesterase inhibitor physostigmine on performance was then assessed in a within-subjects design analyzed using a repeated-measures ANOVA. Physostigmine impaired cognitive flexibility as measured by switches at $0.03 \mathrm{mg} / \mathrm{kg}, F(1,17)=4.7, p$

\section{Training}

Training and testing took place, during weekdays, in 15 operant chambers described previously (Young et al., 2018), consisting of two phases: (1) conditioning magazine illumination with liquid reward delivery $(\sim 30 \mu \mathrm{L}$ strawberry milkshake; Nesquik), delivered every $15 \mathrm{~s}$ for $20 \mathrm{~min}$; (2) instrumental learning wherein mice had to nose poke in one of two apertures to obtain reward. After reaching 70 nose pokes per session for two consecutive sessions in instrumental learning for 4 consecutive days in the habituation phase (Hab2), mice were tested in the PRLT.

\section{Testing}

The PRLT lasted $60 \mathrm{~min}$, wherein the same two stimulus locations during training were presented, but with altered contingencies (see Fig. 1a; Milienne-Petiot et al., 2016). One hole

$<.05$ (b). This effect was driven by physostigmine-induced increases in punishment sensitivity, $F(3,51)=7.6, p<.0001$, at both 0.01 and 0.03 $\mathrm{mg} / \mathrm{kg}(\mathbf{d})$. Only the highest dose slowed latency to choose target stimuli, $F(3,51)=3.4, p<.05(\mathbf{c})$, while no effect of drug was seen on total trials completed; (e), or reward sensitivity as measured by win-stay behavior (f). Data presented as individual data points, mean, \pm SEM. $* p<.05$, as indicated

was designated the target, providing a high probability of reward $(80 \%)$ and low probability $(20 \%)$ of "punishment" (house light illumination for $4 \mathrm{~s}$, indicating the lack of reward). The other (nontarget) hole provided a low probability of reward (20\%) and high probability of punishment $(80 \%)$. Hole-contingencies were reversed after eight consecutive responses at the target hole (quantified as a switch). The number of switches is indicative of executive functioning, while other measures include target win-stay and reward latency (reward sensitivity), lose-shift (punishment sensitivity), mean target response latency (speed of processing), and premature responses (motor impulsivity).

\section{Forced swim test}

Mice were placed into a $4.0 \mathrm{~L}$ beaker filled with $2.5 \mathrm{~L}$ of room temperature $\left(\sim 23.0^{\circ} \mathrm{C}\right)$ water, making it impossible for the animal to touch the bottom of the beaker while keeping its 
head above the water. The test beaker was surrounded on the sides by an opaque box to isolate the animal from the experimenter, who observed behavior by video camera. Immobility (when the animal was not actively swimming and doing the bare minimum to stay afloat) was quantified in total seconds over 5 minutes as described previously (van Enkhuizen et al., 2015). All FST drug treatments were administered in a between-subjects design to prevent habituating effects produced by repeated FST performance.

\section{Experiment 1: Physostigmine-induced effects on punishment-related behaviors in the PRLT}

Adult female and male C57BL6/J mice $(n=10$ and 9, respectively) were acclimated to daily intraperitoneal (i.p.) injections of saline 1 week prior to drug testing to minimize acute stress on test day. On test days, mice were treated with the acetylcholinesterase inhibitor physostigmine sulfate (Sigma-Aldrich, St. Louis, MO, USA), dissolved in $0.9 \%$ saline $(10 \mathrm{ml} / \mathrm{kg})$, and administered i.p. $30 \mathrm{~min}$ prior to behavioral testing $(0.01$, 0.03 , and $0.1 \mathrm{mg} / \mathrm{kg}$ ) in a within-subjects design, with doses chosen based on prior research (van Enkhuizen et al., 2015). A repeated-measures analysis of variance (ANOVA) was used to detect effects of counterbalanced treatments across testing days. The remaining studies were conducted using the FST given the limited efficacy of SAP-inducing punishment sensitivity in healthy animals because of the consistent training times used equates to a strong zeitgeber (Young et al., 2018; Young et al., 2019), whereas FST testing requires one session.

\section{Experiment 2: ACh receptor antagonist effects on physostigmine-induced increase in FST immobility}

Adult male mice ( $n=178 ; n=16-18$ per group) were acclimated to daily i.p. injections of saline 1 week prior to drug testing to minimize acute stress on test day. Thirty minutes prior to FST, mice received either i.p. saline or $0.03 \mathrm{mg} / \mathrm{kg}$ of the AChE inhibitor physostigmine to decrease ACh breakdown. Animals then received a second i.p. injection of the nAChR antagonist mecamylamine (Rabenstein, Caldarone, \& Picciotto, 2006, Sigma, mecamylamine hydrochloride, 0.56 or $0.75 \mathrm{mg} / \mathrm{kg}, 30$ minutes pre-FST), the $\mathrm{mAChR}$ antagonist scopolamine (Witkin et al., 2014, Sigma, scopolamine hydrobromide, 0.03 or $0.065 \mathrm{mg} / \mathrm{kg}, 10 \mathrm{~min}$ pre-FST), or saline (20 min pre-FST). Statistical effects were queried using an ANOVA, with photoperiod and drug treatment as factors. Hypothesized a priori effects of physostigmine were queried using specific one-way ANOVA.
Experiment 3a: $\mathrm{mAChR}$ antagonist effects on shortactive photoperiod-induced increase on immobility

Male $(n=35)$ and female $(n=36)$ mice were initially acclimated to daily i.p. injections of saline 1 week prior to testing. Prior to FST, mice were housed in custom-built photoperiod chambers for 2 weeks under either NA or SA photoperiod conditions. Ten minutes prior to FST, mice received an i.p. injection of either $0.03 \mathrm{mg} / \mathrm{kg}$ scopolamine (Witkin et al., 2014) or saline. Statistical effects were queried using an ANOVA, with sex, photoperiod, and drug treatment as factors. Detecting no interactions with photoperiod or drug, data were collapsed across sex, as depicted in Fig. 3a. Hypothesized a priori effects of scopolamine were queried using a one-way ANOVA.

\section{Experiment 3b: $n A C h R$ antagonist effects on short- active photoperiod-induced increase on immobility}

Male $(n=72)$ and female $(n=72)$ mice were initially acclimated to daily i.p. injections of saline 1 week prior to drug testing. Prior to FST, mice were housed for 2 weeks in either NAP (male $n=36$; female $n=36$ ) or SAP conditions (male $n$ $=36$; female $n=36$ ). Ten minutes prior to FST, mice received an i.p. injection of either $0.56 \mathrm{mg} / \mathrm{kg}$ mecamylamine or saline. This dose of mecamylamine was chosen from our own pilot experiments, which were based on dose ranges tested in Caldarone et al. (2004) and Rabenstein et al. (2006). Statistical effects were queried using an ANOVA, with sex, photoperiod, and drug treatment as factors. Detecting no interactions with photoperiod or drug, data were collapsed across sex, as depicted in Fig. 3b. Hypothesized a priori effects of mecamylamine were queried using a one-way ANOVA.

\section{Experiment 4: Low ineffective doses of $\mathrm{mAChR}$ antagonist and $\mathrm{nAChR}$ antagonist effects on short- active photoperiod-induced increase on immobility}

To further test that both receptor subtypes are contributing to this effect, we sought to test for an additive effect of subthreshold doses of scopolamine and mecamylamine. Male $(n=79)$ and female $(n=79)$ mice were divided into four treatment conditions. Mice were housed in NAP or SAP conditions for 2 weeks prior to testing. One week prior to testing, mice were initially acclimated to daily i.p. injections of saline. Animals always received two i.p. injections administered 30 and 10 minutes prior to FST. The four treatment conditions for the two photoperiods were as follows: (1) saline ( -30 minutes)/ saline (-10 minutes) (SAL/SAL), (2) saline $/ 0.01 \mathrm{mg} / \mathrm{kg}$ scopolamine (SAL/SCP), (3) $0.30 \mathrm{mg} / \mathrm{kg}$ mecamylamine/saline (MEC/SAL), and (4) $0.30 \mathrm{mg} / \mathrm{kg}$ mecamylamine $/ 0.01 \mathrm{mg} / \mathrm{kg}$ scopolamine (MEC/SCP). Drug doses were chosen as 
described above. Statistical effects were queried using an ANOVA, with sex, photoperiod, and drug treatment as factors. Detecting no interactions with photoperiod or drug, data were collapsed across sex as depicted in Fig. 4. Hypothesized a priori effects of drug were queried using a one-way ANOVA.

\section{Experiment 5: Viral expression of human AChE effects on FST immobility in SAP-exposed mice}

Male $(n=44)$ and female $(n=38) \mathrm{C} 57 \mathrm{~B} / 6 \mathrm{~J}$ mice received $2.0 \mu \mathrm{l}$ bilateral infusions (as in Mineur et al., 2013) of either expressing human AChE tagged Adenoassociated Virus2 (AAV2) expressing human AChE tagged with HA for verification of expression (AAV2-CMV-hAChE-HA) or Green Fluorescent Protein (GFP)-reporter only under control of the cytomegalovirus (CMV) promoter at coordinates AP: -2.4 $\mathrm{mm}, \mathrm{ML}: \pm 1.0 \mathrm{~mm}, \mathrm{DV}:-2.0 \mathrm{~mm}$. After 2 weeks postsurgical recovery, mice were moved to NAP or SAP conditions for an additional 2 weeks. FST testing was staggered such that every mouse was tested 14-20 days following surgery over a total of 7 weeks. Mice were excluded from final analysis due to mistargeted injections or undetectable virus expression $(n=$ 16; 10 males, six females). Statistical effects were queried using an ANOVA, with sex, photoperiod, and viral treatment as factors.

At the conclusion of behavioral testing, mice were perfused through the heart with cold saline followed by $4 \%$ paraformaldehyde. The brain was then removed and placed in $4 \%$ paraformaldehyde overnight for postfixation and transferred the following day into $20 \%$ sucrose and kept in this solution until it lost buoyancy. Three sets of $40-\mu \mathrm{m}$ sections were taken through hippocampus and stored in phosphate buffered saline (PBS) with $0.1 \%$ sodium azide until staining. One set of sections was washed $3 \times 5$ minutes with PBS and then $3 \times 5$ minutes in phosphate buffered saline with $0.3 \%$ Triton $\mathrm{X}$ 1000 (PBST). Sections were then incubated in 3\% normal goat serum (NGS; Jackson ImmunoResearch) for 60 minutes. Sections were then incubated overnight in rabbit anti-HA (1:500, Cell Signaling C29F4) in PBST and 3\% NGS. The following day, the tissue was washed $3 \times 5$ minutes in PBST and then transferred into fluorophore-conjugated secondary antibodies for 3 hours. Goat antimouse Alexa Fluor 488 (1:500, Invitrogen 150077) was used to visualize HA-tagged hAChE, and hippocampal morphology was visualized by staining with fluoromount-DAPI. Control virus expression was verified by visualizing GFP.

\section{Statistical analyses}

Data were initially analyzed for homogeneity of variance. After confirmation of normal variance, overall results were analyzed by full-factorial ANOVAs, with sex, drug, photoperiods, and virus as between-subjects factors, while drug was a within-subjects factor for the PRLT, with specific predictions that $0.03 \mathrm{mg} / \mathrm{kg}$ would deleteriously affect performance. Where no interactions with sex were observed, data are shown with both sexes pooled. Significant interactions were followed up with targeted one-way ANOVAs, with Tukey post hoc analyses conducted where applicable. Given that muscarinic and nicotinic antagonists alone decreased immobility on the FST, a priori analyses using specifically targeted one-way ANOVAs were performed in these experiments to determine effects of the antagonists within individual experimental subgroup (e.g., physostigmine vs. vehicle or SAP vs. NAP). All statistical analyses were performed using IBM SPSS Statistics Package, Version 24 (Chicago, Il).

\section{Results}

\section{Experiment 1: Physostigmine increased punishment- sensitive driven cognitive deficits in mice}

Mice treated with physostigmine exhibited numerous differences on behavior in a probabilistic learning task. Primarily, physostigmine increased punish sensitivity as measured by lose-shift behavior on trials following a time-out after choosing the nontarget on the preceding trial (see Fig. 1d), $F(3,51)$ $=7.6, p<.0001$, with both 0.01 and $0.03 \mathrm{mg} / \mathrm{kg}$ increasing lose-shift behavior relative to vehicle $(p<.05)$. Although no main effect of physostigmine was observed on switches, $F(3$, $51)=1.2, p=.33$, the effects of $0.03 \mathrm{mg} / \mathrm{kg}$ was directly compared with vehicle as an a priori hypothesis given that this dose elevated immobility in the FST previously (van Enkhuizen et al., 2015), revealing that this dose significantly lowered the number of switches completed relative to vehicle (see Fig. 1b), $F(1,17)=4.7, p=.046$. No interaction with sex was observed. Physostigmine did not affect target win-stay behavior (see Fig. 1f), $F(3,51)=2.1, p=.117$, or premature responses, $F(3,51)=2.1, p=.117$, data not shown, but the highest dose $(1.0 \mathrm{mg} / \mathrm{kg})$ did slow responding (see Fig. 1c), $F(3,51)=3.4, p=.025$, without affecting reward latency, $F(3$, $51)=0.5, p=.698$, or total trials (see Fig. 1e), $F(3,51)=1.5, p$ $=.215$. Hence, as seen in people with depression (Adida et al., 2011; Must, Horvath, Nemeth, \& Janka, 2013), physostigmine induced an increased sensitivity to punishment and impaired cognitive flexibility.

\section{Experiment 2: $\mathrm{mAChR}$ and $\mathrm{nAChR}$ antagonism attenuated physostigmine-induced FST immobility}

Figure 2 illustrates the average immobility scores for each pretreatment and antagonist treatment group. Overall, physostigmine did not induce a main effect on immobility, $F(1,168)$ $=2.652, p=.105$. AChR antagonist treatment significantly 


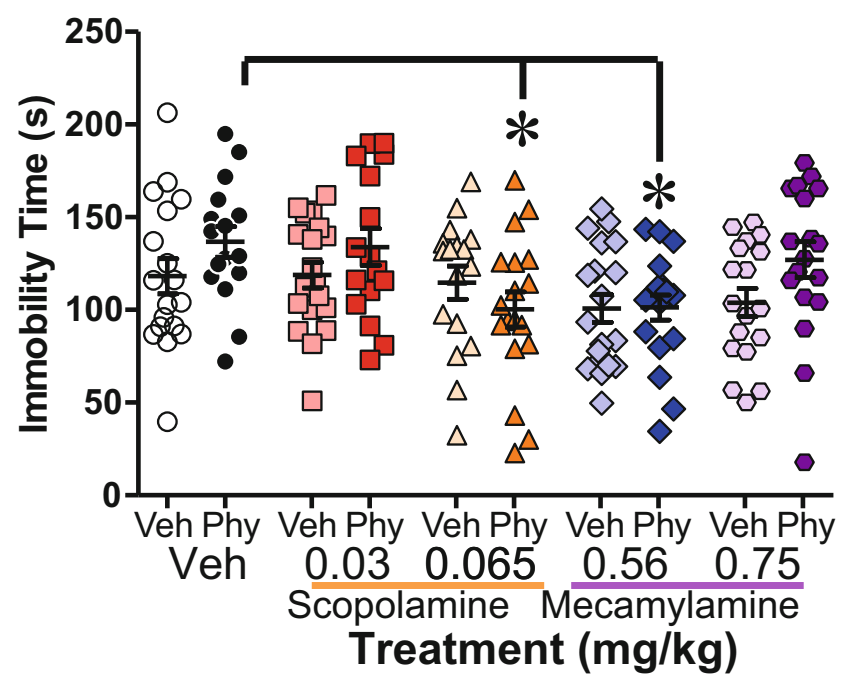

Fig. 2 Both mAChR and nAChR antagonists attenuated physostigmineinduced immobility ( $n=16-18$ male mice per group). Physostigmineinduced increases in forced-swim test immobility was attenuated in animals pretreated with $0.065 \mathrm{mg} / \mathrm{kg}$ of the $\mathrm{mAChR}$ antagonist scopolamine $(\mathrm{SCP}), F(2,47)=5.274, p=.009$, and $0.56 \mathrm{mg} / \mathrm{kg}$ of the nAChR antagonist mecamylamine (MEC), $F(2,53)=4.622, p=.014$. Statistical effects were queried using an ANOVA with photoperiod and drug treatment as factors. Hypothesized a priori effects of physostigmine were queried using a specific one-way ANOVA. Data presented as individual data points, mean, $\pm S E M . * p<.05$, as indicated

decreased FST immobility overall, $F(4,168)=4.037, p=$ .004 , and post hoc analyses revealed that the $0.065 \mathrm{mg} / \mathrm{kg}$ dose of muscarinic antagonist scopolamine and the 0.56 $\mathrm{mg} / \mathrm{kg}$ of nicotinic antagonist mecamylamine significantly reduced immobility versus saline $(p<.05)$. No significant interactions were observed $(F<1, n s)$. Given our a priori hypotheses, we determined whether antagonist treatment produced differential effects in mice pretreated with physostigmine versus saline. In animals pretreated with physostigmine, 0.065 $\mathrm{mg} / \mathrm{kg}$ scopolamine significantly reduced immobility versus saline, $F(2,47)=5.274, p=.009$, while scopolamine exerted no significant effect, $F(2,51)<1$, on animals pretreated with saline. Similarly, $0.56 \mathrm{mg} / \mathrm{kg}$ mecamylamine significantly reduced immobility in physostigmine-treated versus salinetreated mice, $F(2,53)=4.622, p=.014$, but produced no significant effect in saline-pretreated mice, $F(2,52)=1.382$, $p=.260$.

\section{Experiment 3a: $\mathrm{mAChR}$ antagonism attenuated SAP- induced FST immobility}

Figure 3a shows average immobility scores after NAP and SAP exposure and scopolamine treatment. Analyses revealed a significant main effect of sex. $F(1,63)=8.916, p=.004$, with females exhibiting higher levels of immobility. Sex did not interact with any other variable $(F<1, n s)$, indicating no sexually dimorphic responses to photoperiod or drug treatment. SAP significantly increased FST immobility compared with NAP, $F(1,63)=6.492, p=.013$, while scopolamine treatment $(0.03 \mathrm{mg} / \mathrm{kg})$ significantly reduced overall immobility versus vehicle, $F(1,63)=6.894, p=.011$. No significant interaction was detected between any variable. As per our a priori hypotheses, we observed a strong trend of scopolamine reducing immobility in NAP mice $(F=1.681, p=.050)$, with a significant effect observed in SAP mice $(F=1.80, p=.04)$.

\section{Experiment 3b: $n A C h R$ antagonism did not attenuate SAP-induced FST immobility}

Figure $3 \mathrm{~b}$ shows average immobility scores after NAP and SAP exposure and mecamylamine treatment. As in Experiment 2a, a main effect of sex was observed, $F(1,136)$ $=5.970, p=.016$, with higher levels of immobility observed in male mice. Again however, sex did not interact with any other variable $(F<1, n s)$ with no other evidence of sexually dimorphic responses. SAP significantly increased immobility, $F(1,136)=4.320, p=.046$. Mecamylamine $(0.56 \mathrm{mg} / \mathrm{kg})$ treatment did not significantly decrease immobility, $F(1$, $136)=2.002, p=.159$, with no significant interactions or differences in a priori comparisons detected between any variables $(F<1, n s)$.

\section{Experiment 4: Combined treatment with low-dose mAChR and $n A C h R$ antagonists attenuated SAP- induced FST immobility}

Figure 4 depicts the effects of combined low doses of $\mathrm{mAChR}$ and $\mathrm{nAChR}$ antagonists additively reducing SAP induced FST immobility. A strong trend for an effect of sex was detected, $F(1,142)=3.887, p=.051$, with again higher immobility observed in male versus female mice, but no interaction with any other variable $(F<1, n s)$. In this experiment, photoperiod did not significantly affect immobility overall $(F<1$, $n s$ ), but immobility differed significantly between drug treatments, however, $F(3,142)=3.107, p<.05)$ post hoc analysis revealed that immobility was significantly reduced in the $\mathrm{MEC} / \mathrm{SAL}$ and MEC/SCP compared with SAL/SAL-treated mice. No significant interactions were detected between any groups. As per our a priori hypotheses, MEC/SCP significantly reduced immobility in NAP mice, $F(1,37)=2.089, p=.02$, whereas immobility was significantly decreased in SAP mice, $F(3,75)=2.679, p=.026$, in MEC/SAL, $F(1,38)=2.43, p=$ .02 , and MEC/SCP, $F(1,38)=1.88, p=.03$, when compared with SAL/SAL treated mice.

\section{Experiment 5: Exogenous expression of $\mathrm{hAChE}$ in hippocampus blocked SAP-induced FST immobility}

Expression of HA-tagged hAChE and control GFP in the hippocampus is shown in Fig. 5, as is the effect of treatment. Of the 82 mice tested in this experiment, two were excluded from 


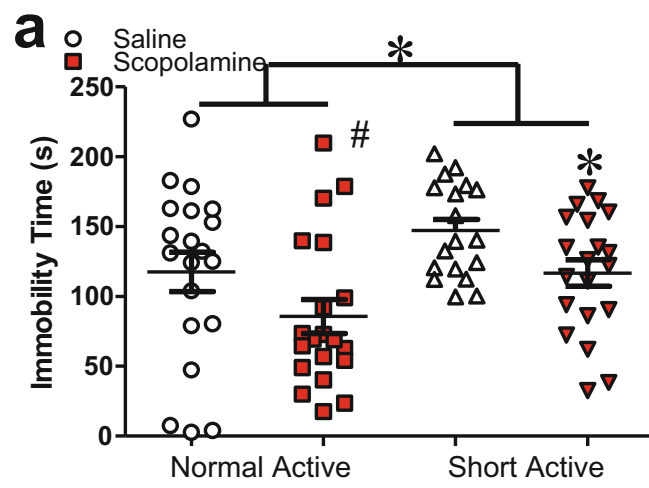

Fig. 3 ACh antagonists attenuated SAP-induced FST immobility. Elevated forced swim test (FST) immobility induced by 2-week exposure to short active photoperiod was blocked by treatment with $0.03 \mathrm{mg} / \mathrm{kg}$ scopolamine. Short active photoperiod significantly increased FST immobility $F(1,63)=6.492, p=.013(\mathbf{a}), F(1,136)=4.320, p=.046(\mathbf{b})$, which was significantly attenuated by $\mathrm{mAChR}$ antagonist scopolamine,

final analysis due to mistargeted injections, and 14 in which no viral expression was evident. In total, the final analysis included 32 female and 34 male mice. A significant Virus $\times$ Photoperiod interaction was observed, $F(1,58)=11.034, p<$ .005 , with hAChE reducing immobility selectively in SAP mice. In contrast, hAChE expression significantly increased immobility in NAP mice. A significant Sex $\times$ Photoperiod interaction was also detected, $F(1,58)=4.255, p<.05$. for

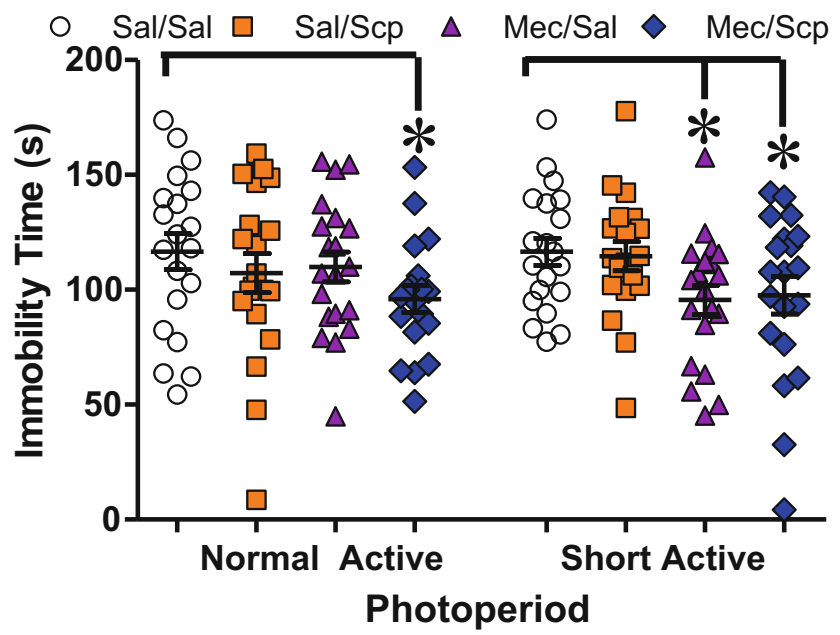

Fig. 4 Low-dose mecamylamine alone blocked SAP-induced FST immobility while only a combined dose is effective in NAP mice. Combined low doses of both nAChR and mAChR selectively attenuate immobility in short active photoperiod (SAP) mice. In NAP mice the combined dose significantly decreased immobility, $F(1,37)=2.089, p=.02$. In SAP mice, $0.30 \mathrm{mg} / \mathrm{kg}$ mecamylamine significantly attenuated immobility, $F(3,75)=2.679, p=.026$, in the forced swim test (FST), both alone, $F(1,38)=2.43, p=.02$, and combined with low-dose $0.01 \mathrm{mg} / \mathrm{kg}$ scopolamine, $F(1,38)=1.88, p=.03$. Data presented as individual data points, mean, $\pm S E M . * p<.05$ where indicated. Statistical effects were queried using ANOVA with sex, photoperiod, and drug treatment as factors. Detecting no interactions with photoperiod or drug, data was collapsed across sex as depicted in Fig. 4. Hypothesized a priori effects of drug were queried using one-way ANOVA

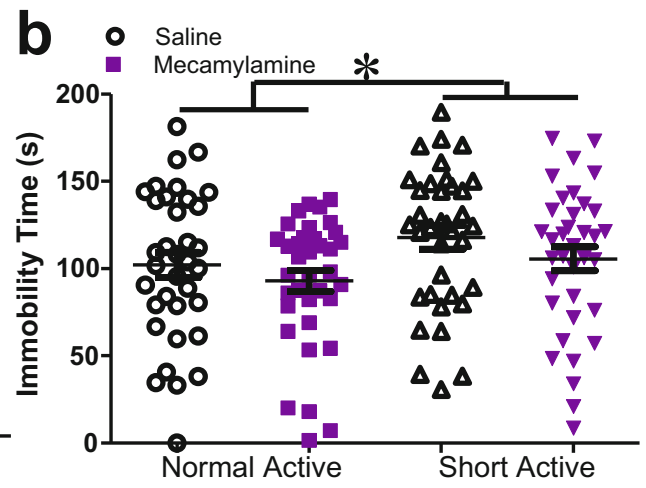

$F(1,136)=1.80, p=.04$ (a). The $\mathrm{nAChR}$ antagonist mecamylamine did not reduce immobility at $0.56 \mathrm{mg} / \mathrm{kg}$ dose in this experiment. Statistical effects in these data were queried using ANOVA with, photoperiod, and drug treatment as factors. Data presented as individual data points, mean, \pm SEM. $\# p<.10 . * p<.05$

the first time, with female NAP housed mice exhibiting higher levels of immobility compared with all other groups $(p<.05$ compared with female-SA, $p<.05$ compared with male-NA, $p$ $<.05$ compared with male-SA). Post hoc results for the full factorial comparisons indicated this effect was driven by elevated immobility in hAChE mice, which was greater in females compared with males, though no Sex $\times$ Photoperiod $\times$ Virus interaction was observed. Increased immobility resulting from hAChE injection in NAP mice was evident in both sexes. Overall, these data indicate that supplementing $\mathrm{AChE}$ enzymatic breakdown of $\mathrm{ACh}$ in the hippocampus blocks SAP-induced FST immobility.

\section{Discussion}

These results provide evidence of a role for cholinergic mechanisms in driving FST immobility in response to short active photoperiods (SAP). Pharmacological blockade of ACh hydrolysis (via physostigmine administration) increased immobility in the FST, consistent with previous studies in mice, and elevated punishment sensitivity in the PRLT, in concordance with effects measured by clinical rating scales and behavioral tests in humans. Consistent with the idea that elevated $\mathrm{ACh}$ was responsible for this stress-induced behavior that has been used to model behavioral phenotypes relevant to depression (Mineur et al., 2013), pharmacological blockade of cholinergic transmission of both mAChRs and nAChRs attenuated this physostigmine-induced increase in FST immobility. Similar effects were recapitulated in animals exposed to SAP. Further, increased expression of hAChE in the hippocampus attenuated SAP-induced increase in immobility, suggesting that increased ACh normally could mediate this behavioral response to light, or at least alleviate the homeostatic perturbations induced by SAP, whether it involves ACh 

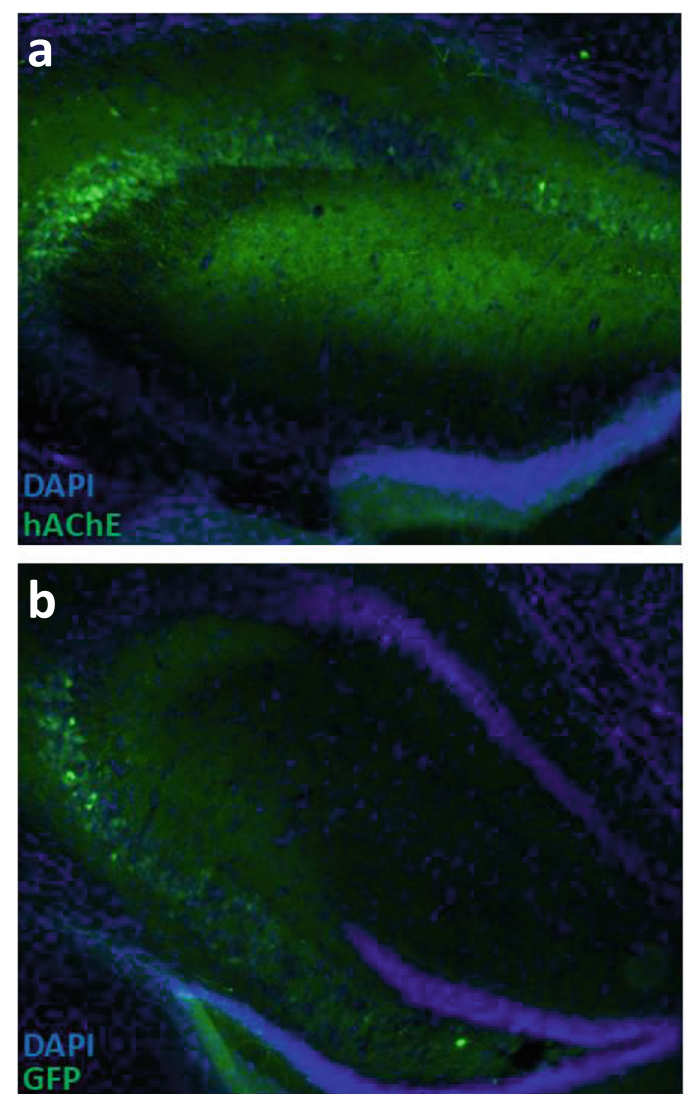

Fig. 5 Vector delivered hAChE blocked the expression of SAP-induced FST immobility. Exogenous expression of human Acetylcholinesterase (hAChE; Alexa Goat-Anti Rabbit 488, Green; a) resulting from bilateral injection of CMV-AAV2 into the hippocampus (outlined by DAPI, Blue) produced a significant interaction with photoperiod, $F(1,58)=11.034, p$ $=.002$, with significantly reduced immobility in short active photoperiod (SAP) housed mice compared with controls (GFP; b). Immobility was

directly or not. These data support our overarching hypotheses that SAP elevates ACh, a mechanism relevant to the precipitation of depression in humans, and leads to increased FST immobility in mice, and these phenotypes can be remediated via blockade of AChRs.

This work helps define the role of ACh circuitry underlying the induction of depression by seasonal factors, furthering our understanding of the mechanisms of SAD and $\mathrm{BD}$ depression (Desvignes et al., 2003; Janowsky et al., 1973; Janowsky et al., 1972). Specifically, the AChE inhibitor physostigmine increased depressed states in people, which has been recapitulated in rodents (Mineur et al., 2013), and can be blocked by the mood stabilizer lithium (van Enkhuizen et al., 2015). Further, scopolamine has demonstrated efficacy as a rapidacting antidepressant in clinical trials (Drevets, Zarate, \& Furey, 2013; Furey \& Drevets, 2006; Furey et al., 2013). The present studies provide further support for the reproducibility of these physostigmine findings and potential downstream mechanisms of its action, as the $0.03 \mathrm{mg} / \mathrm{kg}$ dose here and in van Enkhuizen (2015) repeatedly increased FST immobility. Decreases in locomotion have been reported for

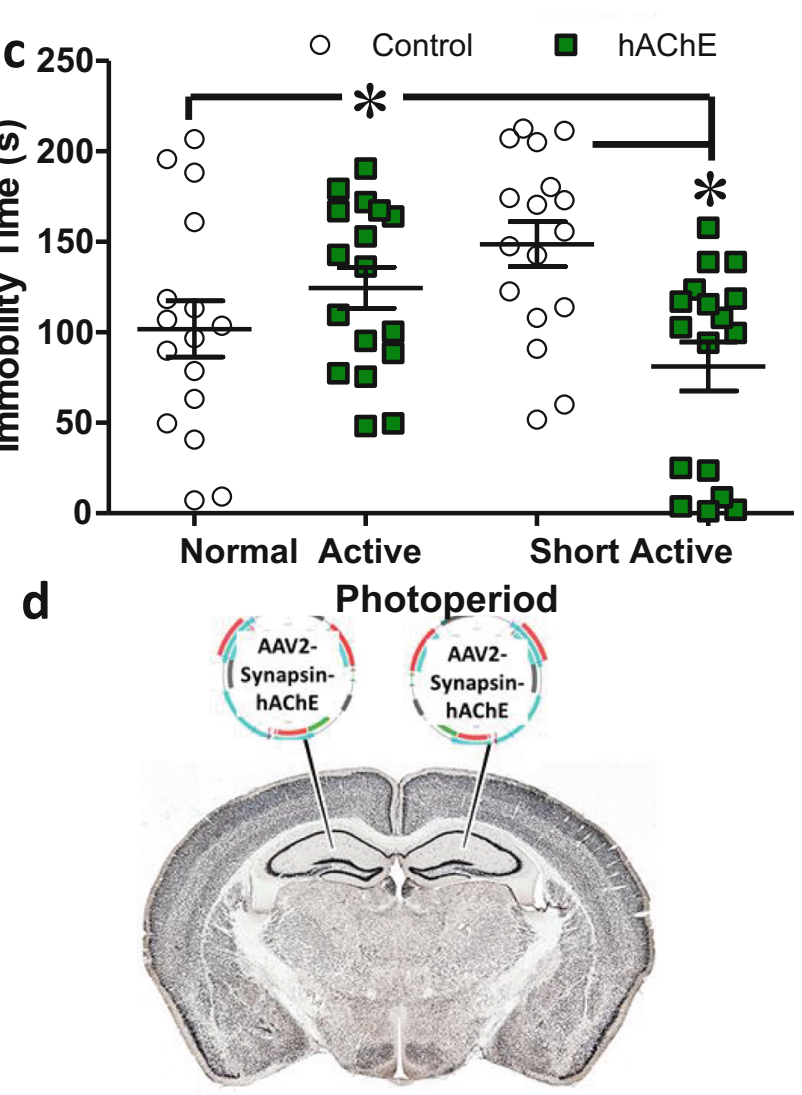

also increased in hAChE-treated mice housed under a normal active photoperiod (c). Placement of bilateral injections represented in schematic (d). Data presented as individual data points, mean, $\pm S E M$. $* p<.05$, as indicated. Statistical effects were queried using ANOVA with sex, photoperiod, and viral treatment as factors. Absent a significant three-way interaction with sex, data shown are collapsed across this variable

treatment with $0.03 \mathrm{mg} / \mathrm{kg}$ physostigmine in mice (Mach, Grubbs, Price, Paton, \& Lucot, 2004). However, in our previous experiments, this dose did not decrease locomotor activity (van Enkhuizen et al., 2015), nor did it affect mean target or reward latency in the PRLT presented here, suggesting that these effects do not result from locomotor suppression. Consistent with our hypotheses, both $\mathrm{mAChR}$ and $\mathrm{nAChR}$ blockade reduced immobility selectively in animals pretreated with physostigmine. Notably, knockdown of alpha7 nAChRs within the hippocampus has been shown to attenuate responses to physostigmine (Mineur et al., 2018). These studies support the idea that ACh signaling via both $\mathrm{mAChRs}$ and nAChRs likely mediate depressive-like behaviors, certainly those induced via physostigmine.

Given the hypersensitivity of people with BD to seasonally-induced depression (Geoffroy et al., 2015), we also conducted parallel studies to determine the ACh contribution to SAP-induced FST immobility. We hypothesized that SAP would induce FST immobility mediated via nAChR and mAChRs. Animals housed under the SAP condition exhibited increased FST immobility, which was blocked by 
scopolamine (see Fig. 3a). Mecamylamine treatment decreased SAP-induced immobility similarly to scopolamine, although this effect was not significant. We had predicted that a low dose of the mecamylamine or scopolamine separately would be insufficient to reduce elevated FST immobility, but that their combination would produce a significant reduction in SAP-induced immobility, not individually. Both mecamylamine alone, and as well as the mecamylamine and scopolamine combination, significantly reduced immobility, however (see Fig. 4). Although immobility in the NAP and SAP groups did not differ significantly, it is worth noting that the low-dose mecamylamine decreased immobility selectively in animals exposed to the SAP only, indicative of alterations within nAChR signaling pathways specific to SAP exposure. Therefore, while treatment with nAChR antagonists can decrease SAP immobility, the effect may be sensitive to differences in receptor dynamics induced by SAP not yet understood.

Finally, we demonstrated that exogenous expression of hAChE in the hippocampus could reduce immobility selectively in SAP mice. This observation aligns with the hypothesis that SAP-induced immobility is a result of increased ACh signaling in this brain region. Further, SAP also increased punishment sensitivity in mice (Young et al., 2018), highlighting the potential clinical relevance of this mechanism in seasonal BD and SAD. Cholinergic activity within nucleus accumbens and ventral tegmental area can also mediate reward sensitivity (Collins, Aitken, Greenfield, Ostlund, \& Wassum, 2016; Small, Nunes, Hughley, \& Addy, 2016; Wickham, Solecki, Rathbun, McIntosh, \& Addy, 2013) as well as FST immobility (Addy, Nunes, \& Wickham, 2015), indicating an important interplay within these highly connected pathways. The mechanistic studies here were conducted using the FST given the in-the-moment sensitivity of this task. Training in the PRLT limits the impact of SAP, as genetic hypersensitivity (e.g., DAT-HT mice) is required to see baseline effects (Young et al., 2018). Hence, the mechanistic studies were conducted in the FST. Determining the role of these pathways in aberrant systems such as after SAP exposure could identify additional targets for treating depression-relevant symptoms.

The effect of hAChE increasing immobility in NA exposed mice in Experiment 5 was unexpected, and especially prominent in female mice. Regardless of sex, this effect may be a result of depletion of ACh in these animals, resulting in divergent effects compared with SAP mice, which would be expected to have higher levels of ACh signaling based on these studies. Additionally, sex effects were inconsistent in presentation and direction across the four experiments, highlighting the likelihood that other factors influencing this relationship were not fully captured here. Given the sex differences seen in presentation of SAD and depression (Azorin, Adida, \& Belzeaux, 2015; Borisnekov et al., 2015), in addition to the FST, future studies will fully include sex. Nor were other seasonally relevant factors accounted for, such as temperature, light spectrum, or food availability, which may modulate depression-related behaviors both naturalistically and in the lab setting. Here, we only modulated the amount of light exposure. These natural factors are difficult to mimic in a laboratory setting. This belies our reliance on FST in this set of experiments. FST can be completed in a single trial and does not require mice to leave their photoperiod chambers at any point before the experiment. In contrast, PRLT requires daily training with highly palatable rewards, which can act as extraneous zeitgeibers polluting SAP effects. Future experiments accounting for these factors are warranted.

Our findings indicate the important mediating role of $\mathrm{mAChR}$ and $\mathrm{nAChRs}$ underlying depressive-like behaviors. More imperatively, our results demonstrate that the same ACh pathways likely play a role in the underlying mechanism of depressive-like behavior resulting from SAP. This work supports our hypothesized model of the ACh circuitry underlying $\mathrm{SAD}$ and seasonal $\mathrm{BD}$, whereby SAP would drive expression of somatostatin in the neurons of the paraventricular hypothalamic nucleus; this in turn increases CRF (Dulcis et al., 2013; Young et al., 2018), which drives hippocampal ACh to produce depressive-like behavior. Our previous finding supported the SAP and SST link (Young et al., 2018), the SAP and CRF link (Dulcis et al., 2013), and here, the final supporting factor that ACh plays in this pathway likely initiated by elevated CRF (Desvignes et al., 2003). Future studies will focus on behaviors used in those studies relevant to the amotivation seen in people with depression (Young \& Dulcis 2015). Numerous neuropharmacological, neuroimaging, and genetic studies on BD conclude that an increase of catecholamines such as dopamine and norepinephrine contribute to mania symptoms, while an elevation of ACh levels contribute to depressive symptoms (Hannestad et al., 2013; Higley \& Picciotto, 2014; van Enkhuizen et al., 2015; Young \& Dulcis, 2015). As this study focused on the depressive state of $\mathrm{BD} / \mathrm{SAD}$, our findings support the elevation of cholinergic transmission in this state, consistent with previous research regarding the catecholamine-choline balance hypothesis of BD (van Enkhuizen et al., 2015).

In summary, we provide evidence that implicates winterlike photoperiod (SAP) exposure inducing elevated hippocampal ACh, driving depression-relevant behaviors. Systemic ACh elevation via treatment with the AChE inhibitor physostigmine increased punish-sensitivity behavior and immobility. Physostigmine-induced immobility was blocked by systemic $\mathrm{nAChR}$ and $\mathrm{mAChR}$ antagonists. SAP recreated this profile, which was similarly blocked by these antagonists. Importantly, regional sufficiency of elevated ACh for this SAP-induced effect was demonstrated such that intrahippocampal infusions of human AChE also attenuated SAP effects. While our previous evidence demonstrates that SAP elevates Somatostatin (SST) and CRF in the PVN of 
mice (Young et al., 2018) and rats (Dulcis et al., 2013), and decreases Tyrosine hydroxylase (TH) neurons in the midbrains of humans (Aumann et al., 2016), here, we make clear that hippocampal $\mathrm{ACh}$, acting via $\mathrm{nAChR}$ and $\mathrm{mAChR}$, is an important end effector of SAP-induced FST immobility. Delineating the connections between elevated SST and CRF to hippocampal ACh, as well as the specific subtypes of receptors responsible for depression-relevant behaviors more directly in translatable behaviors remains vital if more targeted therapeutics are to be discovered.

Acknowledgements We would like to thank Dr. Mark A. Geyer, Ms. Mahalah R. Buell, and Mr. Richard F. Sharp for their support throughout this work. These studies were made possible by funding from NIMH Grant R01MH104344-03, R01DA043535, as well as by the Veteran's Administration VISN 22 Mental Illness Research, Education, and Clinical Center.

Author contributions Z.C., M.L., A.J., C.v.d.C., and M.K. conducted all the behavioral experiments originally designed by Z.C., D.D., and J.Y. with support from M.P. and Y.M. Z.C., J.L., and A.H. conducted the postmortem brain imaging analyses. Z.C. and J.Y. wrote the initial manuscript, with support and editing from all authors.

Funding and disclosures Dr. Young has received grant support from Sunovion Pharmaceuticals for work unrelated to the above manuscript. All authors report funding from NIH, but no potential conflict.

\section{References}

Addy, N. A., Nunes, E. J., \& Wickham, R. J. (2015). Ventral tegmental area cholinergic mechanisms mediate behavioral responses in the forced swim test. Behavioural Brain Research, 288, 54-62. https://doi.org/10.1016/j.bbr.2015.04.002

Adida, M., Jollant, F., Clark, L., Besnier, N., Guillaume, S., Kaladjian, A., ... Courtet, P. (2011). Trait-related decision-making impairment in the three phases of bipolar disorder. Biological Psychiatry, 70, 357-65. https://doi.org/10.1016/j.biopsych.2011.01.018.

Amitai, N., Young, J. W., Higa, K., Sharp, R. F., Geyer M. A., \& Powell S. B. (2014). Isolation rearing effects on probabilistic learning and cognitive flexibility in rats. Cognitive, Affective, \& Behavioral Neuroscience, 14, 388-406.

Ashkenazy-Frolinger, T., Kronfeld-Schor, N., Juetten, J., \& Einat, H. (2010). It is darkness and not light: Depression-like behaviors of diurnal unstriped Nile grass rats maintained under a short photoperiod schedule. Journal of Neuroscience Methods, 186(2), 165-170.

Aumann, T. D., Raabus, M., Tomas, D., Prijanto, A., Churilov, L., Spitzer, N.C., ... Horne, M. K. (2016). Differences in number of midbrain dopamine neurons associated with summer and winter photoperiods in humans. PLOS ONE, 11(7), e0158847. https://doi. org/10.1371/journal.pone.0158847

Azorin, J. M., Adida, M., \& Belzeaux, R. (2015) Frequency and characteristics of individuals with seasonal pattern among depressive patients attending primary care in France. General Hospital Psychiatry, 37(1), 76-80.

Barbini, B., Di Molfetta, D., Gasperini, M., Manfredonia, M., \& Smeraldi, E. (1995). Seasonal concordance of recurrence in mood disorder patients. European Psychiatry, 10(4), 171-174.

Borisnekov, M. F., Petrova, N. B., Timonin, V. D, Fradkova, L. I., Kolomeichuk, S. N., Losova, A. L., \& Kasyanova, O. N. (2015).
Sleep characteristics, chronotype and winter depression in 10-20year olds in northern European Russia. Journal of Sleep Research, 24(3), 288-295.

Caldarone, B. J., Harrist, A., Cleary M. A., Beech, R. D., King, S. L., \& Picciotto, M. R. (2004). High-affinity nicotinic acetylcholine receptors are required for antidepressant effects of amitriptyline on behavior and hippocampal cell proliferation. Biological Psychiatry, 56(9), 657-664.

Collins, A. L., Aitken, T. J., Greenfield, V. Y., Ostlund, S. B., \& Wassum, K. M. (2016). Nucleus accumbens acetylcholine receptors modulate dopamine and motivation. Neuropsychopharmacology, 41(12), 2830-2838.

Desvignes, C,, Rouquier, L,, Souilhac, J,, Mons, G,, Rodier, D,, Soubrie, P., \& Steinberg, R. (2003). Control by tachykinin NK(2) receptors of CRF(1) receptor-mediated activation of hippocampal acetylcholine release in the rat and guinea-pig. Neuropeptides, 37(2), 89-97.

Drevets, W. C., Zarate, C. A., \& Furey, M. L. (2013). Antidepressant effects of the muscarinic cholinergic receptor antagonist scopolamine: A review. Biological Psychiatry, 73(12), 1156-1163

Dulcis, D., Jamshidi, P., Leutgeb, S., \& Spitzer, N. C. (2013). Neurotransmitter switching in the adult brain regulates behavior. Science, 340(6131), 449-453.

Fernandez, D. C., Fogerson, P. M., Lazzerini Ospri, L., Thomsen, M. B., Layne, R. M., Severin, D, ... Hattar, S. (2018). Light affects mood and learning through distinct retina-brain pathways. Cell 175(1), $71-84$.

Furey, M. L., \& Drevets, W. C. (2006). Antidepressant efficacy of the antimuscarinic drug scopolamine: A randomized, placebocontrolled clinical trial. Archives of General Psychiatry, 63(10), 1121-1129.

Furey M. L., Drevets, W. C., Hoffman, E. M., Frankel, E., Speer, A. M., \& Zarate, C. A. (2013). Potential of pretreatment neural activity in the visual cortex during emotional processing to predict treatment response to scopolamine in major depressive disorder. JAMA Psychiatry. 70(3):280-290

Geoffroy, P. A., Lajnef, M., Bellivier, F., Jamain, S., Gard, S., Kahn, J. P., ... Etain, B. (2015). Genetic association study of circadian genes with seasonal pattern in bipolar disorders. Scientific Reports, 5, 10232. https://doi.org/10.1038/srep10232

Hannestad, J. O., Cosgrove, K. P., DellaGioia, N. F., Perkins, E., Bois, F., Bhagwagar, Z., Seibyl, J. P., ... Esterlis, I. (2013). Changes in the cholinergic system between bipolar depression and euthymia as measured with [ $\left.{ }^{123} \mathrm{I}\right]$ 5IA single photon emission computed tomography. Biological Psychiatry, 74(10, ) 768-776.

Higley, M. J., \& Picciotto, M. R. (2014). Neuromodulation by acetylcholine: Examples from schizophrenia and depression. Current Opinion in Neurobiology, 29, 88-95.

Janowsky, D. S., el-Yousef, K., Davis, J. M., \& Sekerke, H. J. (1973). Parasympathetic suppression of manic symptoms by physostigmine. Archives of General Psychiatry, 28(4), 542-547.

Janowsky, D. S., el-Yousef, M. K., Davis, J. M., Hubbard, B., Sekerke, H. J. (1972). Cholinergic reversal of manic symptoms. Lancet, 1(7762), 1236-1237.

Lee, H. C., Tsai, S. Y., \& Lin, H. C. (2007). Seasonal variations in bipolar disorder admissions and the association with climate: A populationbased study. Journal of Affective Disorders, 97(1/3), 61-69.

Mach, M., Grubbs, R. D., Price, W. A., Paton, S. J., \& Lucot, J. B. (2004). Behavioral changes after acetylcholinesterase inhibition with physostigmine in mice. Pharmacology Biochemistry and Behavior, 79(3), 533-540.

Milienne-Petiot, M., Kesby, J. P., Graves, M., van Enkhuizen, J., Semenova, S., Minassian, A., ... Young, J. W. (2016). The effects of reduced dopamine transporter function and chronic lithium on motivation, probabilistic learning, and neurochemistry in mice: Modeling bipolar mania. Neuropharmacology, 113(Pt. A), 260270 . 
Milienne-Petiot, M., Higa, KK., Grim, A., Deben, D., Groenink, L., Twamley, EW., ... Young, JW. (2018). Nicotine improves probabilistic reward learning in wildtype but not alpha7 $\mathrm{nAChR}$ null mutants, yet alpha 7 agonists do not improve probabilistic learning. European Neuropsychopharmacology, 28(11):1217-1231

Mineur, Y. S., Obayemi, A., Wigestrand, M. B., Fote, G. M., Calarco, C. A., Li, A. M., \& Picciotto, M. R. (2013). Cholinergic signaling in the hippocampus regulates social stress resilience and anxiety- and depression-like behavior. Proceedings of the National Academy of Sciences of the United States of America, 110(9) 3573-3578.

Mineur, YS., Mose, TN., Blakeman, S., Picciotto, MR., (2018). Hippocampal alpha7 nicotinic ACh receptors contribute to modulation of depression-like behavior in C57BL/6J mice. British Journal of Pharmacology. 175(11):1903-1914.

Mulder, R. T., Cosgriff, J. P., Smith, A. M., \& Joyce, P. R. (1990). Seasonality of mania in New Zealand. Australian and New Zealand Journal of Psychiatry, 24(2), 187-190.

Must, A., Horvath, S., Nemeth, V. L., \& Janka, Z. (2013). The Iowa gambling task in depression-What have we learned about suboptimal decision-making strategies? Frontiers in Psychology, 4, 732.

Parker, G., \& Walter, S. (1982). Seasonal variation in depressive disorders and suicidal deaths in New South Wales. British Journal of Psychiatry, 140, 626-632.

Parry, B. L., \& Maurer, E. L. (2003). Light treatment of mood disorders. Dialogues in Clinical Neuroscience, 5(4), 353-365.

Partonen, T., \& Lonnqvist, J. (1998). Seasonal affective disorder. Lancet 352(9137), 1369-1374.

Postolache, T. T., \& Oren, D. A. (2005). Circadian phase shifting, alerting, and antidepressant effects of bright light treatment. Clinics in Sports Medicine, 24(2), 381-413, xii.

Rabenstein, R. L., Caldarone, B. J., \& Picciotto, M. R. (2006). The nicotinic antagonist mecamylamine has antidepressant-like effects in wild-type but not $\beta 2$ - or $\alpha 7$-nicotinic acetylcholine receptor subunit knockout mice. Psychopharmacology, 189, 395-401.

Risch, S. C., Cohen, R. M., Janowsky, D. S., Kalin, N. H., Sitaram, N., Gillin, J. C., \& Murphy, D. L. (1981). Physostigmine induction of depressive symptomatology in normal human subjects. Psychiatry Research, 4(1), 89-94. https://doi.org/10.1016/0165-1781(81) 90012-3

Rosenthal, N. E., Sack, D. A., Gillin, J. C., Lewy, A. J., Goodwin, F. K., Davenport, Y., ... Wehr, T. A. (1984). Seasonal affective disorder: A description of the syndrome and preliminary findings with light therapy. Archives of General Psychiatry, 41(1), 72-80.

Saricicek. A, Esterlis. I, Maloney, K. H., Mineur, Y.S., Ruf, B.M., Muralidharan, A., ... Bhagwagar, Z. (2013). Persistent beta2*-nicotinic acetylcholinergic receptor dysfunction in major depressive disorder. American Journal of Psychiatry, 169, 851-859

Sayer, H. K., Marshall, S., Mellsop, G. W. (1991). Mania and seasonality in the southern hemisphere. Journal of Affective Disorders, 23(3), $151-156$.
Shopsin, B., Janowsky, D., Davis, J., \& Gershon, S. (1975). Rebound phenomena in manic patients following physostigmine: Preliminary observations. Neuropsychobiology, 1(3), 180-187.

Small, K. M., Nunes, E., Hughley, S., \& Addy, N. A. (2016). Ventral tegmental area muscarinic receptors modulate depression and anxiety-related behaviors in rats. Neuroscience Letters, 616, 80 85. https://doi.org/10.1016/j.neulet.2016.01.057

Takei, N., O'Callaghan, E., Sham, P., Glover, G., Tamura, A., \& Murray, R. (1992). Seasonality of admissions in the psychoses: Effect of diagnosis, sex, and age at onset. British Journal of Psychiatry, 161, 506-511.

van Enkhuizen, J., Milienne-Petiot, M., Geyer, M. A., \& Young, J. W. (2015). Modeling bipolar disorder in mice by increasing acetylcholine or dopamine: Chronic lithium treats most, but not all features. Psychopharmacology (Berl), 232(18), 3455-3467.

Volpe, F. M., \& Del Porto, J. A. (2006). Seasonality of admissions for mania in a psychiatric hospital of Belo Horizonte, Brazil. Journal of Affective Disorders, 94(1/3), 243-248.

Wang, B., \& Chen, D. (2013). Evidence for seasonal mania: A review. Journal of Psychiatric Practice, 19(4), 301-308. https://doi.org/10. 1097/01.pra.0000432600.32384.c5

Wickham, R., Solecki, W., Rathbun, L., McIntosh, J. M., \& Addy, N. A. (2013). Ventral tegmental area alpha6beta2 nicotinic acetylcholine receptors modulate phasic dopamine release in the nucleus accumbens core. Psychopharmacology (Berl), 229(1), 73-82.

Witkin, J. M., Overshiner, C., Li, X., Catlow, J. T., Wishart, G. N., Schober, D. A., ... Felder, C. C. (2014). M1 and M2 muscarinic receptor subtypes regulate antidepressant-like effects of the rapidly acting antidepressant scopolamine. Journal of Pharmacology and Experimental Therapeutics, 351(2), 448-456.

Wu, J. C., Kelsoe, J. R., Schachat, C., Bunney, B. G., DeModena, A., Golshan, S., ... Bunney, W. E. (2009). Rapid and sustained antidepressant response with sleep deprivation and chronotherapy in bipolar disorder. Biological Psychiatry, 66(3), 298-301. https://doi.org/ 10.1016/j.biopsych.2009.02.018

Young, J. W., \& Dulcis, D. (2015). Investigating the mechanism(s) underlying switching between states in bipolar disorder. European Journal of Pharmacology, 759, 151-162.

Young, J. W., Cope, Z. A., Romoli, B., Schrurs, E., Aniek, J., van Enkhuizen, J., ... Dulcis, D. (2018). Mice with reduced DAT levels recreate seasonal-induced switching between states in bipolar disorder. Neuropsychopharmacology, 43, 1721-1731. https://doi.org/10. 1038/s41386-018-0031-y

Young, J. W., Cope, Z. A., Romoli, B., Schrurs, E., Joosen, A., van Enkhuizen, J., ... Dulcis, D. (2019). Evidence for lightentrainment-induced switching between depression- \& maniarelevant behaviors in mice. Neuropsychopharmacol, 44, 16791680 .

Publisher's note Springer Nature remains neutral with regard to jurisdictional claims in published maps and institutional affiliations. 